\title{
COVID-19 can affect the heart
}

\author{
By Eric J. Topol \\ Scripps Research Translational Institute, Scripps Research, La Jolla, CA, USA. Email: etopol@scripps.edu
}

\section{COVID-19 has a spectrum of potential heart manifestations with diverse mechanisms}

The family of seven known human coronaviruses are known for their impact on the respiratory tract, not the heart. However, the most recent coronavirus, severe acute respiratory syndrome coronavirus 2 (SARS-CoV-2), has marked tropism for the heart and can lead to myocarditis (inflammation of the heart), necrosis of its cells, mimicking of a heart attack, arrhythmias, and acute or protracted heart failure (muscle dysfunction). These complications, which at times are the only features of coronavirus disease 2019 (COVID-19) clinical presentation, have occurred even in cases with mild symptoms and in people who did not experience any symptoms. Recent findings of heart involvement in young athletes, including sudden death, have raised concerns about the current limits of our knowledge and potentially high risk and occult prevalence of COVID-19 heart manifestations.

The four "common cold" human coronaviruses-HCoV229E, HCoV-NL63, HCoV-OC43, and HCoV-HKU1-have not been associated with heart abnormalities. There were isolated reports of patients with Middle East respiratory syndrome (MERS; caused by MERS-CoV) with myocarditis and a limited number of case series of cardiac disease in patients with SARS (caused by SARS-CoV) (1). Therefore, a distinct feature of SARS-CoV-2 is its more extensive cardiac involvement, which may also be a consequence of the pandemic and the exposure of tens of millions of people to the virus.

What appears to structurally differentiate SARS-CoV-2 from SARS is a furin polybasic site that, when cleaved, broadens the types of cells (tropism) that the virus can infect (2). The virus targets the angiotensin-converting enzyme 2 (ACE2) receptor throughout the body, facilitating cell entry by way of its spike protein, along with the cooperation of the cellular serine protease transmembrane protease serine 2 (TMPRSS2), heparan sulfate, and other proteases (3). The heart is one of the many organs with high expression of ACE2. Moreover, the affinity of SARS-CoV-2 to ACE2 is significantly greater than that of SARS (4). The tropism to other organs beyond the lungs has been studied from autopsy specimens: SARS-CoV-2 genomic RNA was highest in the lungs, but the heart, kidney, and liver also showed substantial amounts, and copies of the virus were detected in the heart from 16 of 22 patients who died (5). In an autopsy series of 39 patients dying from COVID-19, the virus was not detectable in the myocardium in $38 \%$ of patients, whereas $31 \%$ had a high viral load above 1000 copies in the heart (6).

Accordingly, SARS-CoV-2 infection can damage the heart both directly and indirectly (see the figure). SARS-CoV-2 exhibited a striking ability to infect cardiomyocytes derived from induced pluripotent stem cells (iPSCs) in vitro, leading to a distinctive pattern of heart muscle cell fragmentation, with "complete dissolution of the contractile machinery" (7). Some of these findings were verified from patient autopsy specimens. In another iPSC study, SARS-CoV-2 infection led to apoptosis and cessation of beating within 72 hours of exposure (8). Besides directly infecting heart muscle cells, viral entry has been documented in the endothelial cells that line the blood vessels to the heart and multiple vascular beds. A secondary immune response to the infected heart and endothelial cells (endothelitis) is just one dimension of many potential indirect effects. These include dysregulation of the renin-angiotensin-aldosterone system that modulates blood pressure, and activation of a proinflammatory response involving platelets, neutrophils, macrophages, and lymphocytes, with release of cytokines and a prothrombotic state. A propensity for clotting, both in the microvasculature and large vessels, has been reported in multiple autopsy series and in young COVID-19 patients with strokes.

There is a diverse spectrum of cardiovascular manifestations, ranging from limited necrosis of heart cells (causing injury), to myocarditis, to cardiogenic shock (an often fatal inability to pump sufficient blood). Cardiac injury, as reflected by concentrations of troponin (a cardiac muscle-specific enzyme) in the blood, is common with COVID-19, occurring in at least one in five hospitalized patients and more than half of those with preexisting heart conditions. Such myocardial injury is a risk factor for in-hospital mortality and troponin concentration correlates with risk of mortality. Furthermore, patients with higher troponin amounts have markers of increased inflammation [including C-reactive protein, interleukin-6 (IL-6), ferritin, lactate dehydrogenase (LDH), and high neutrophil count] and heart dysfunction (amino-terminal pro-B-type natriuretic peptide) (9).

More worrisome than the pattern of limited injury is myocarditis: diffuse inflammation of the heart, usually representing a variable admixture of injury and the inflammatory 
response to the injury that can extend throughout the three layers of the human heart to the pericardium (which surrounds the heart). Unlike SARS-associated myocarditis, which did not exhibit lymphocyte infiltration, this immune and inflammatory response is a typical finding at autopsy after SARS-CoV-2 infections. Involvement of myocytes, which orchestrate electrical conduction, can result in conduction block and malignant ventricular arrhythmias, both of which can lead to car-diac arrest.

Along with such in-hospital arrythmias, there have been reports of increased out-of-hospital cardiac arrest and sudden death in multiple geographic regions of high COVID-19 spread, such as the $77 \%$ increase in Lombardy, Italy, compared with the prior year (10). There have been many reports of myocarditis simulating a heart attack, owing to the cluster of chest pain symptoms, an abnormal electrocardiogram, and increased cardiac-specific enzymes in the blood, even in patients as young as a 16-year-old boy. When there is extensive and diffuse heart muscle damage, heart failure, acute cor pulmonale (right heart failure and possible pulmonary emboli), and cardiogenic shock can occur.

COVID-19-associated heart dysfunction can also be attributed to other pathways, including Takotsubo syndrome (also called stress cardiomyopathy), ischemia from endothelitis and related atherosclerotic plaque rupture with thrombosis, and the multisystem inflammatory syndrome of children (MIS-C). The underlying mechanism of stress cardiomyopathy is poorly understood but has markedly increased during the pandemic. MIS-C is thought to be immune-mediated and manifests with a spectrum of cardiovascular features, including vasculitis, coronary artery aneurysms, and cardiogenic shock. This syndrome is not exclusive to children because the same clinical features have been the subject of case reports in adults, such as in a 45 year old man (11).

Recent series of COVID-19 patients undergoing magnetic resonance imaging (MRI) or echocardiography of the heart have provided some new insights about cardiac involvement (12-14). In a cohort of 100 patients recovered from COVID-19, 78 had cardiac abnormalities, including 12 of 18 patients without any symptoms, and 60 had ongoing myocardial inflammation, which is consistent with myocarditis (12). The majority of more than 1200 patients in a large prospective cohort with COVID-19 had echocardiographic abnormalities (13). This raises concerns about whether there is far more prevalent heart involvement than has been anticipated, especially because at least 30 to $40 \%$ of SARS-CoV-2 infections occur without symptoms. Such individuals may have underlying cardiac pathology.

To date, there have been four small series of asymptomatic individuals with bona fide infections who underwent chest computed tomography (CT) scans to determine whether there were lung abnormalities consistent with
COVID-19. Indeed, half of the asymptomatic people showed lung CT features that were seen in patients with symptoms. But so far, there have been minimal cardiac imaging studies in people who test positive for SARS-CoV-2 or are seropositive but without symptoms. Furthermore, the time course of resolution or persistence of any organ abnormalities after SARSCoV-2 infection has not yet been reported. With a high proportion of silent infections despite concurrent evidence of internal organ damage, there is a fundamental and large hole in our knowledge base.

In contrast to people without symptoms, there is a substantial proportion of people who suffer a longstanding, often debilitating illness, called long-COVID. Typical symptoms include fatigue, difficulty in breathing, chest pain, and abnormal heart rhythm. An immunologic basis is likely but has yet to be determined. Nor have such patients undergone systematic cardiovascular assessment for possible myocarditis or other heart abnormalities, such as fibrosis, which could account for some of the enduring symptoms. It would not be surprising in the future for patients to present with cardiomyopathy of unknown etiology and test positive for SARSCoV-2 antibodies. However, attributing such cardiomyopathy to the virus may be difficult given the high prevalence of infections, and ultimately a biopsy might be necessary to identify virus particles to support causality.

Cardiac involvement in athletes has further elevated the concerns. A 27-year-old professional basketball player, recovered from COVID-19, experienced sudden death during training. Several college athletes have been found to have myocarditis (14), including 4 of $26(15 \%)$ in a prospective study from Ohio State University (15), along with one of major league baseball's top pitchers. Collectively, these young, healthy individuals had mild COVID-19 but were subsequently found to have unsuspected cardiac pathology. This same demographic group-young and healthy-are the most common to lack symptoms after SARS-CoV-2 infections, which raises the question of how many athletes have occult cardiac disease? Systematic assessment of athletes who test positive for SARS-CoV-2, irrespective of symptoms, with suitable controls through some form of cardiac imaging and arrhythmia screening seems prudent until more is understood.

The most intriguing question that arises is why do certain individuals have a propensity for heart involvement after SARS-CoV-2 infection? Once recognized a few months into the pandemic, the expectation was that cardiac involvement would chiefly occur in patients with severe COVID-19. Clearly, it is more common than anticipated, but the true incidence is unknown. It is vital to determine what drives this pathogenesis. Whether it represents an individual's inflammatory response, an autoimmune phenomenon, or some other explanation needs to be clarified. Beyond preventing SARS-CoV-2 infections, the goal of averting cardiovascular 
involvement is paramount. The marked heterogeneity of COVID-19, ranging from lack of symptoms to fatality, is poorly understood. A newly emerged virus, widely circulating throughout the human population, with a panoply of disease manifestations, all too often occult, has made this especially daunting to unravel.

\section{REFERENCES AND NOTES}

1. T. Y. Xiong, S. Redwood, B. Prendergast, M. Chen, Eur. Heart J. 41, 1798 (2020). doi:10.1093/eurhearti/ehaa231 Medline

2. N. J. Matheson, P. J. Lehner, Science 369, 510 (2020). doi:10.1126/science. abc6156 Medline

3. F. Hikmet et al., Mol. Syst. Biol. 16, e9610 (2020). doi:10.15252/msb.20209610 Medline

4. A. Gupta et al., Nat. Med. 26, 1017 (2020). doi:10.1038/s41591-020-0968-3 Medline

5. V. G. Puelles et al., N. Engl. J. Med. 383, 590 (2020). doi:10.1056/NEJMc2011400 Medline

6. D. Lindner et al., JAMA Cardiol. 10.1001/jamacardio.2020.3551 (2020).

7. J. A. Perez-Bermejo et al., bioRxiv 265561 [Preprint] 12 September 2020); doi:10.1101/2020.08.25.265561.

8. A. Sharma et al., Cell Rep. Med. 10.1016/j.xcrm.2020.100052 (2020).

9. R. O. Bonow, G. C. Fonarow, P. T. O'Gara, C. W. Yancy, JAMA Cardiol. 5, 751 (2020). doi:10.1001/jamacardio.2020.1105 Medline

10. E. Baldi et al.; Lombardia CARe Researchers, N. Engl. J. Med. 383, 496 (2020). doi:10.1056/NEJMc2010418 Medline

11. S. Shaigany et al., Lancet 396, e8 (2020). doi:10.1016/S0140-6736(20)31526-9 Medline

12. V. O. D. Puntmann et al., JAMA Cardiol. 10.1001/jamacardio.2020.3557 (2020).

13. M. Dwecket al., Eur. Heart J. 10.1093/ehjci/jeaa178 (2020).

14. L. Huang et al., JACC Cardiovasc. Imaging 10.1016/j.jcmg.2020.05.004 (2020).

15. S. Rajpal et al., JAMA Cardiol. 10.1001/jamacardio.2020.4916 (2020).

\section{ACKNOWLEDGMENTS}

E.J.T. is supported by National Institutes of Health grant UL1 TR001114.

Published online 23 September 2020

10.1126/science.abe2813 


\section{Damaging the heart}

Severe acute respiratory syndrome coronavirus 2 (SARS-CoV-2) infection has the potential to directly and indirectly induce cardiac damage.

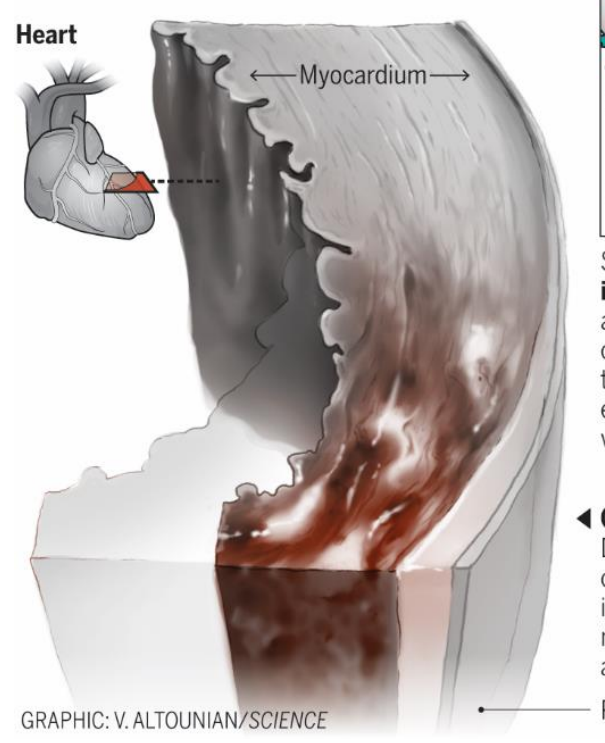

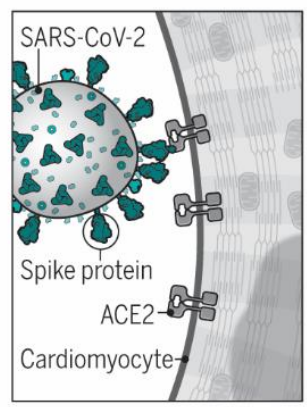

SARS-CoV-2 can directly infect cardiomyocytes, attaching to angiotensinconverting enzyme 2 (ACE2) through its spike protein and entering the cells by fusing viral and cellular membranes.

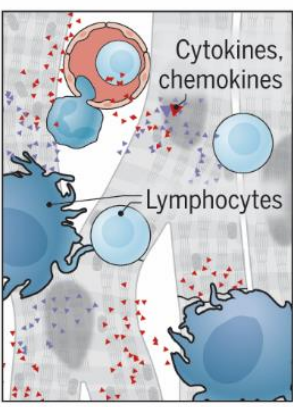

SARS-CoV-2 infection can indirectly damage cardiomyocytes through systemic inflammatory responses and diminished blood supply (e.g., from blood clots and endothelitis, not shown)

Complications

Damaged cardiomyocytes, necrosis, and cardiogenic shock can result from direct and/or indirect effects of SARS-CoV-2 infection. This can lead to scarring and thinning of the myocardium, myocarditis, cardiomyopathy, arrhythmias, and potentially cardiac arrest.

Pericardium 